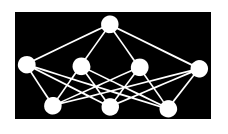

\title{
FRACTAL DIMENSION AS A DESCRIPTOR OF URBAN GROWTH DYNAMICS
}

\author{
Pavel Tuček, and Zbyněk Janoška $a^{\dagger}$
}

\begin{abstract}
The objective of this paper is to examine the development of the urban form of the city of Olomouc since the 1920s in terms of fractal dimension, and to link the observation with two other descriptors of shape - area and perimeter. The fractal dimension of built-up areas and fractal dimension of the boundary of the city are calculated employing the box-counting method; the possibilities of their interpretation and usage in urban planning are discussed. The process of urban growth is observed with respect to its fractality and perspectives of this approach are discussed. An interesting dependence between area and its fractal dimension is derived.
\end{abstract}

Key words: Urban growth, fractal dimension, box-counting method, area/perimeter relation

Received: September 20, 2011

Revised and accepted: February 14, 2013

\section{Introduction}

Since Mandelbrot's famous paper entitled by How Long Is the Coast of Britain? Statistical Self-Similarity and Fractional Dimension [10], a fractal dimension is widely recognized as a valuable tool for description of shape. It has gained a large popularity in the geosciences [1,9] (among other disciplines), where measures of object shape are essential. The year 1994 can be considered as the beginning of the exploration of cities in terms of fractal geometry; in this year, the book by Batty \& Longley called Fractal Cities: A Geometry of Form and Function was published [1], being currently regarded as a classic. They presented a number of ways in which fractal geometry (and, especially, fractal dimension) can be used for examining the form of a city; here, we will follow their work in a certain way.

\footnotetext{
* Pavel Tuček

Department of Geoinformatics, Palacký University in Olomouc, Svobody 26, 77146 Olomouc, Czech Republic, and Department of Mathematical Analysis and Applications of Mathematics, Faculty of Science, Palacký University in Olomouc, 17. listopadu 12, 77146 Olomouc, Czech Republic, E-mail: pavel.tucek@upol.cz

†Zbyněk Janoška

Department of Geoinformatics, Faculty of Science, Palacký University in Olomouc, Svobody 26, 77146 Olomouc, Czech Republic, E-mail: zbynek.janoska@centrum.cz
} 
Indeed, urban patterns display a fractal behavior. Similar structures emerging in different scales (compare the global network of roads and highways with a city and its transportation network) can be observed [3], however, it is obvious that patterns observed on a local scale are not exact copies of the patterns observed on a global scale. Cities can be viewed as a complex system consisting of smaller parts, each of them embodying a pattern of its own. We may observe a self-similar structure in both suburbs and blocks of prefab buildings, however, they are not the same in principle.

Based on this observation, Myint warns that the usage of fractal geometry for description of cities as a whole may lead to considerable confusion [14]. Another problem emerges from the calculation of fractal dimension. Since real-world objects can not be described in a mathematical way, there is no exact formula for calculating the fractal dimension. There are many approaches how to estimate fractal dimension, each giving, more or less, different results. Thus, one must pick the appropriate technique carefully [16].

In this paper, we focus on the issue of change in the urban form of the city of Olomouc, Czech Republic. Detailed aerial imagery covering almost 80 years are available. The fractal dimension of both boundary and area occupied by the city has been observed and the underlying causes of such behavior have been studied in detail. Up to this point, Batty [1], as well as other researchers, are followed. Relations between fractal dimension, area and perimeter of the urban form are further examined and possibilities of using those relations for future predictions of urban growth and for urban planning are then discussed.

The structure of the paper is as follows. In Section 2, we expand the description of the concept of fractality and how fractal dimension is estimated. In Section 3, we describe data assembly and processing and in Section 4, we present results obtained. Various aspects regarding quality of data, their interpretation and other issues are discussed in Section 5. We conclude with final remarks and prospects of future work in Section 6 .

\section{Methodology}

\subsection{Fractal dimension}

The concept of fractality has been described in detail in many works $[7,9,11,15$, 17]. Fractal dimension is a measure of complexity of shape, based on irregularity, scale dependence and self-similarily of objects [5]. Fractal objects repeat the same structure in all scales, making them self-similar and giving them a scale-invariant property [19]. Many real-world objects show this behavior [11], such as clouds, coastlines, vascular systems, fern leaves, etc. The basic property of all fractal structures is their dimension. Although there is no exact definition of fractals, the widely accepted definition comes from Mandelbrot himself: "A fractal is by definition a set for which the Hausdorff-Besicovitch dimension strictly exceeds the topological dimension" [11]. The Hausdorff-Besicovitch dimension is thus a number, which describes the complexity of an object and its value is non-integral. The bigger the value of the Hausdorff-Besicovitch dimension, the more complex the shape of the object is, thus filling more space. The Hausdorff-Besicovitch dimension is commonly known as a fractal dimension. 
Fractal dimension is a measure of the complexity of a shape, however, it is independent of area or perimeter of the object. Two objects with the same area or perimeter can have completely different fractal dimensions. This theoretical notion is a crucial point, because, as we will show later, there exists a relation between area occupied by the city and its fractal dimension. Chen [4] shows that some indices, commonly used to describe urban settlements, can be directly derived from fractal direction; however, he does not explore specifically the relation of fractal dimension to area or perimeter.

As shown in [1], fractals have infinite perimeter and finite area. Therefore it seems pointless to explore the perimeter of the city in terms of fractal dimension. However, attempt to find a relation among area, perimeter, fractal dimension of developed areas and fractal dimension of the city boundary is made in this paper.

As has already been mentioned above, there is a number of methods to estimate the fractal dimension and, as [16] points out, results obtained by various methods often differ significantly. Not only the method itself, but a software, which calculates the fractal dimension, may contribute to the differences as well [16]. In the presented study, we use Fractalyse, a free software easily obtainable from http://www.fractalyse.org/.

\subsection{The box-counting method}

The box-counting method to estimate the fractal dimension was used for experimental data being in the form of binary pictures. The box-counting dimension of a set $S$ contained in ${ }^{n}$ is defined as follows: For any $\varepsilon>0$, let $N_{\varepsilon}(S)$ be the minimum number of $n$-dimensional cubes of side length $\varepsilon$ needed to cover $S$. If there is a number $d$, providing that

$$
N_{\varepsilon}(S) \sim \frac{1}{\varepsilon^{d}} \text { as } \varepsilon \rightarrow 0,
$$

we say that a box-counting dimension of $S$ is $d$ [18]. Dimension $d$ can be computed as $[18]$

$$
d=-\lim _{\varepsilon \rightarrow 0} \frac{\ln N_{\varepsilon}(S)}{\ln \varepsilon} .
$$

Calculation of the box-counting dimension is simple. For a sequence of cell sizes $\varepsilon>0$, the number of cells $N_{\varepsilon}(S)$ needed to cover the set $S$ is calculated. The box-counting dimension $d$ can be estimated by the slope of the straight line formed by plotting $\ln \left(N_{\varepsilon}(S)\right)$ against $\ln (\varepsilon)$ (known also as the Richardson-Mandelbrot plot [6]). If the trend is linear, the observed object is assumed to be fractal [8].

\section{Data Processing}

In our case study, we focus on the city of Olomouc. Olomouc city is rather typical in many aspects. Having its center on a small hill above the Morava river and surrounded by a flat land, it does not have natural boundaries at all and urban growth is, therefore, not limited by georelief. Olomouc has been a historical center of Moravia and, therefore, it was of vital importance for the rulers of the Kingdom 


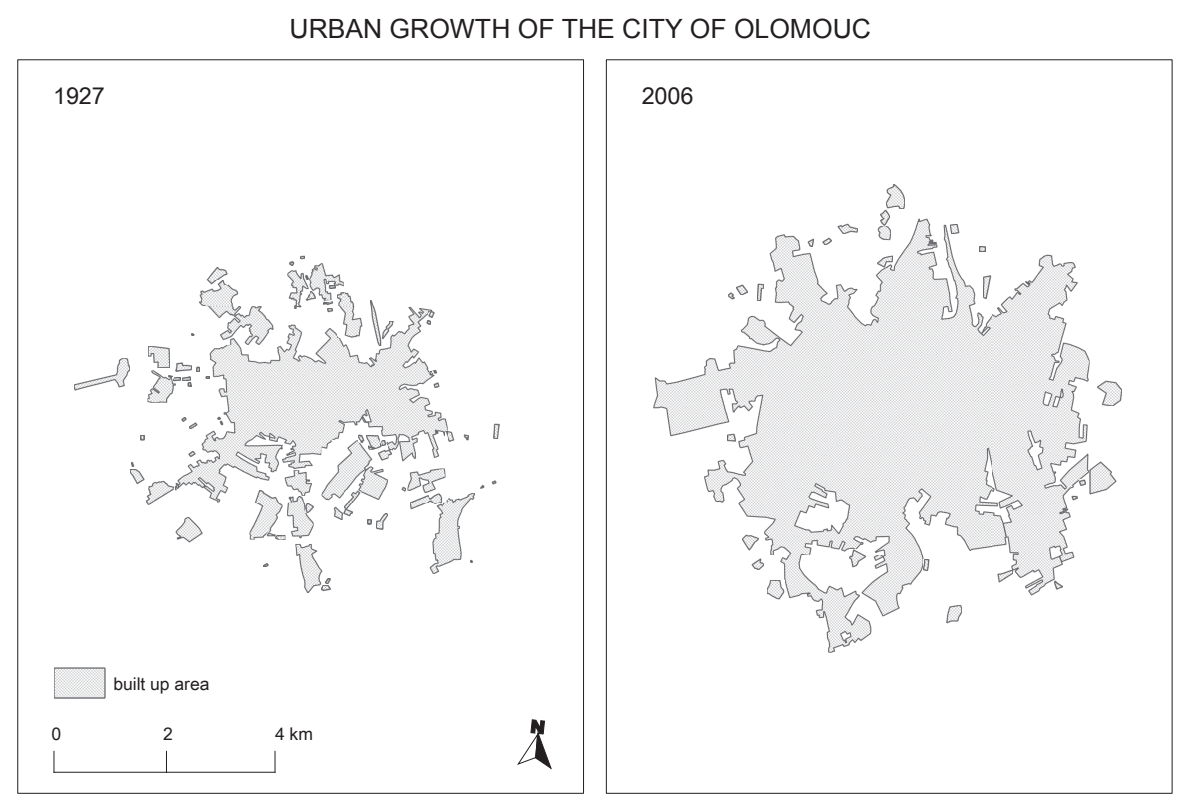

Fig. 1 Urban form of Olomouc in 1927 (left) and 2006 (right).

of Bohemia to keep the city safe. Olomouc was heavily fortified from the $16^{\text {th }}$ to the $19^{\text {th }}$ century and since the law had prohibited building new houses in the neighborhood of the city walls, the city kept its shape until 1886, when its walls were torn down.

Growth of the city is observed to occur in several phases during the $20^{\text {th }}$ century. At first, small villages near Olomouc grew close to the city. Consequently, the historical city had been extended by a belt of semi-attached houses and villas. From the 1950s to the 1980s, blocks of prefab houses grew rapidly at the edge of the city and surrounding villages were joined with the growing city.

In the 1990s, the fabrication of panel houses stopped and most recently, suburbs and large warehouses are being built at the edges of the city.

Aerial imageries from the years 1926, 1971, 1978, 1991, 2001, 2003 and 2006 were used in the paper to identify the boundary of the city.

While it is hard to find any distinguished structure in the urban form in 1927, the resemblance of the urban form to the Koch curve (Fig. 2 left) is eminent in 2006. The star-like shape of the city is not a coincidence, for the Olomouc bastion fortress was inspired by the Renaissance ideal city. The Koch snowflake has a fractal dimension equal to 1.262 [1], thus, it would only seem logical for the boundary of Olomouc to have similar fractal dimension.

The historical center of Olomouc has been chosen as a study area and those villages and settlements which eventually became parts of the city were also included in the analysis. The size of the study area has an indispensable influence on the 
fractal dimension as it defines the space in which the city grows.

As pointed out earlier, the single largest compact developed area was not the only area included in the analysis as the surrounding of this central area was examined as well. It might not seem like a problem in later years, when the central area forms more than $95 \%$ of all built-up area; however, in 1927, the central area contributed only $64 \%$ to the overall built-up area. Both the central city and its surroundings were considered as our study area, since the urbanization process of Olomouc city was heavily influenced by its surroundings. It is evident from Fig. 1 that the development was realized primarily in directions of former surrounding villages; it is therefore necessary to include those settlements in the calculation.

The question of size will be discussed further in the discussion section, together with its possible impact on the results.
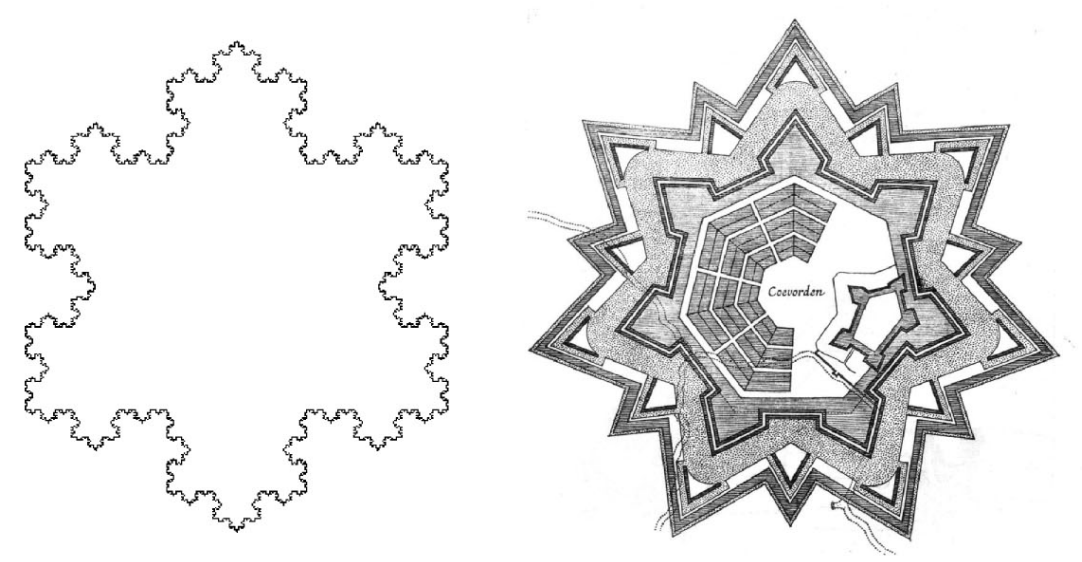

Fig. 2 Koch curve [13] (left) and star fort [2] (right).

When studying historical maps, applying the fractal dimension is not a new approach at all. A long list of studies is available, showing that the fractal dimension of urban forms grows with time. The fractal dimension of London increased from 1.332 to 1.791 between 1820 and 1962 and the fractal dimension of Berlin increased from 1.43 to 1.69 between 1875 and 1945 [16]. A common problem associated with such studies is that only few measurements are available. Studies, when more than three timestamps are available, are rare and usually they do not exploit any fractal measures [12]. In this study, seven timestamps are available, which constitutes a reasonable sample to estimate long-term trends. Not only the values of fractal dimension of Olomouc will be observed, but it is more important to examine the relation between the fractal dimension and other descriptors of shape, i.e. area and perimeter. Fractal objects do not have a finite perimeter but they do have a finite area. Thus, we suppose that there will be a relation between fractal dimension and area, but none between fractal dimension and perimeter. 
Both absolute values of fractal dimension and their relations with time, area and perimeter will be examined. Found dependencies will be interpreted and their significance towards urban planning will be discussed.

\section{Results}

The fractal dimension of both built-up areas and their boundary were measured together with the area and perimeter. The results are presented in Tab. I.

\begin{tabular}{ccccc}
\hline Year & $\begin{array}{c}\text { Area } \\
\left(\mathrm{m}^{2}\right)\end{array}$ & $D_{\text {Area }}$ & $\begin{array}{c}\text { Perimeter } \\
(\mathrm{km})\end{array}$ & $D_{\text {Perimeter }}$ \\
\hline 1927 & 10.485 & 1.683 & 106.57 & 1.363 \\
1971 & 21.188 & 1.758 & 116.61 & 1.284 \\
1978 & 23.780 & 1.780 & 117.14 & 1.285 \\
1991 & 28.028 & 1.808 & 108.12 & 1.268 \\
2001 & 28.726 & 1.813 & 112.29 & 1.274 \\
2003 & 29.064 & 1.816 & 111.28 & 1.272 \\
2006 & 29.639 & 1.816 & 112.36 & 1.272 \\
\hline
\end{tabular}

Tab. I Descriptors of the shape of Olomouc.

It is obvious that both area and its fractal dimension grow constantly with time. Perimeter, and its fractal dimension, on the other hand, does not seem to have a strict trend. This would only support the hypothesis presented in the previous section. Plotted values are presented in Figs. 3 and 4.
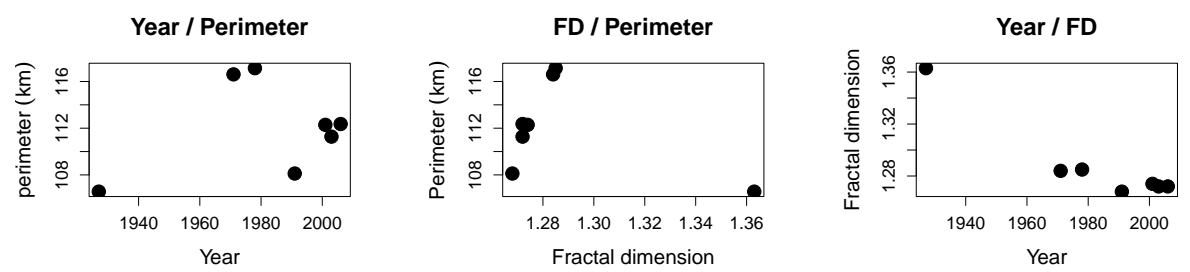

Fig. 3 Relation between time and perimeter (left), time and fractal dimension (middle) and perimeter and fractal dimension (right).

\subsection{Perimeter/fractal dimension relation}

It seems that the fractal dimension of the perimeter of the city tends to cluster around value of 1.27 , which is a sound confirmation of the previously stated resemblance to the Koch curve with a fractal dimension of 1.26. Apart from this, no other structure can be observed in Fig. 3. This corresponds with the theoretical notion of absence of finite perimeter of fractal objects. 

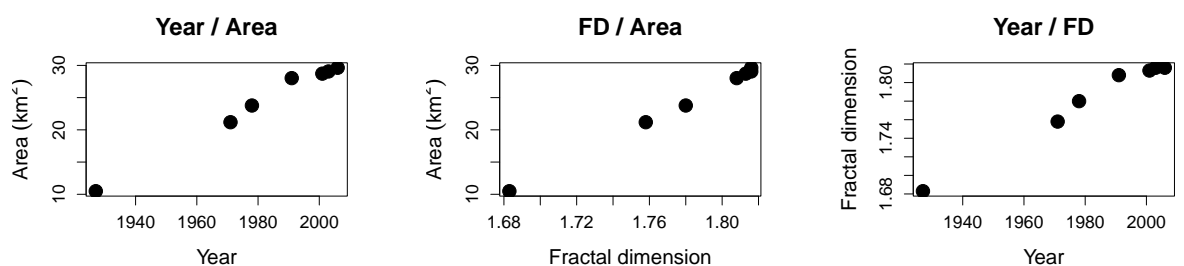

Fig. 4 Relation between time and area (left), time and fractal dimension (middle) and area and fractal dimension (right).

\subsection{Area/fractal dimension relation}

As can be seen, the relation between area and its fractal dimension is strictly linear. We can thus construct a linear regression model to describe this relation. This model has the form of

$$
\text { Fractal Dimension }=1.609103+0.007081 \times \text { Area } .
$$

As mentioned above, the fractal dimension is a measure of complexity of shape. It is equal to 1 for a straight line, 2 for a circle or a square, 3 for a cube or sphere etc. For a real-world object in a plane, it has values larger than 1 and lower than 2 . Although cities have more complex shapes with a vertical dimension as well, their cartographical representation is planar and, therefore, they are usually examined as planar objects.

A plane has a fractal dimension equal to 2. From the observed trend, it is obvious that eventually the growing area will have a fractal dimension equal to 2 ; the city will fill up the whole plane.

By a simple calculation, the fractal dimension of 2 would correspond to an area of $55.2 \mathrm{~km}^{2}$.

Does this mean that this area is critical for the city of Olomouc? Nowadays, the built-up area is $29.6 \mathrm{~km}^{2}$, slightly more than a half of the predicted value. Thus, we can only guess what the future development will be. At this place, we can suggest two possible explanations, considering the hypothesis of linear relation between area and its fractal dimension:

1. The predicted area of $55.2 \mathrm{~km}^{2}$ is a limit which cannot be reached; the city will never grow up to this size.

2. Once the city reaches the predicted area of $55.2 \mathrm{~km}^{2}$, it will continue to grow. However, since its complexity in plane cannot grow any longer, the growth will be realized in the vertical dimension, resulting in the building of high-rise buildings.

The important fact is that while the distance from the center of Olomouc to its peripheries is approximately $4 \mathrm{~km}$, a circle with a volume of $55.2 \mathrm{~km}^{2}$ has a radius of $4.2 \mathrm{~km}$. Therefore, a natural interpretation would be to expect Olomouc to grow only to "fill" the undeveloped areas within the distance of $4.2 \mathrm{~km}$ from the city center. 
Looking back on Year/Area and Year/Fractal Dimension relations depicted in Fig. 4, it can be seen that although the trend as a whole seems to be linear, when the value for 1927 is cut off, the trend can be considered parabolic. This would point to a slowing process of growth of the area of the city. Unfortunately, there are not enough data to confirm the hypothesis and, therefore, no final conclusions can be made.

If the results made in the previous section are taken into account, namely the observation of fractal dimension of boundary clustering around the value of 1.27 , it is clear that the city may never reach the state in which it would fully occupy the space with a fractal dimension of 2 . Since a planar object with a dimension equal to 2 can never have its boundary with a dimension equal to 1.27 , the logical explanation would be that the city will never grow to the state where it would occupy an area of $55.2 \mathrm{~km}^{2}$.

\section{Discussion}

The fact that fractal dimension is a valid descriptor of urban growth is well known [1]. The presented study shows that the fractal dimension can be used not only to examine the growth of the city in the past, but may also be a tool to help predict the future development of the city. The relation between built-up area and fractal dimension of an urban form is suggested as linear. This is important as in the last 80 years, there have been different strategies employed in urban planning; however, the relation between area and fractal dimension has remained the same.

The predicted area of the city of Olomouc was also calculated. This area should correspond to the fractal dimension of 2 to satisfy the area/fractal dimension relation. However, no city has been reported to have a fractal dimension equal to 2 . The highest reported value is the fractal dimension of Beijing - 1.96 [8].

Moreover, the urban growth is realized not only in a plane, but also in a vertical dimension, which is difficult to incorporate into current analyses.

Fractal dimension could be a helpful tool for urban planners, since it gives an easily interpretable descriptor of complexity of an urban form. However, the possibilities for prediction are limited. Even if it would be possible to predict the fractal dimension and area corresponding with a certain time, it would be impossible to predict the exact areas of the city where the urban growth would be realized.

One option would be to run several simulations (using, for example, cellular automata), and to choose the one which gives the best results with respect to predicted area and fractal dimension.

We are aware that our results were obtained by examining only one city and may not be applicable to other cities, especially to cities with different geographical conditions and historical development. On the other hand, growing fractal dimension through time was observed all over the world, and we believe that the area/fractal dimension relation we presented is of universal nature.

Since the urbanization process is still in progress, the question is whether the study area should not be larger. There are other settlements on the edge of the city, which are certainly influenced by the urban form of Olomouc. However, it is difficult to distinguish between areas which have an active influence on the urban 
form of Olomouc and those which are only influenced by it. Only those areas, which eventually became parts of the city, are taken into account in the analysis.

If study area would be enlarged, the fractal dimension of built-up areas would be smaller, because the city would not fill the space as well as it does now, and new settlements would cover up the study area, resulting thus in a more shattered pattern. The fractal dimension of the city boundary should, on the other hand, remain the same.

Our results and conclusions on maximum area which can be occupied by the city is, therefore, applicable only in an idealized world, where there are no other settlements around the city. Since there are some in our case study, the city of Olomouc spatially develops in their direction and they are eventually connected to it. Nevertheless, our results are a good example of how spatial processes can be described in terms of the fractal dimension and how those descriptors can be used to view the city not as a series of different states in time, but as a changing system with its own dynamics. Perhaps in geographical areas with sparse settlement, our results could be applied in a better way, since the city would not be influenced by its surroundings to a larger extent. Reaching a state where the city would completely fill the space (for example, circle-shaped) would also be logical with respect to minimization of costs.

\section{Conclusion and Future Work}

Changes of fractal dimension in the urban form of the city of Olomouc was observed. The relations between built-up area, perimeter and their fractal dimension were examined. A dependence between built-up area and its fractal dimension emerged, which may lead to interesting assumptions on future development of the city. Consequently, the fractal dimension of the boundary of the city seems to cluster around the value of 1.27 , which is close to the value of the Koch curve.

Possible interpretations of the found dependencies were presented and these interpretations were briefly discussed from the viewpoint of urban planning. The readers are encouraged to view presented results in a critical way. Although these results are consistent in their logic, their application is limited, because the growth of a city is always influenced by external processes and development that would lead to an equilibrium, as presented here is not possible.

In the future, we would like to focus on the functional aspect of cities and their parts, and how they are related. Some studies suggest that fractal dimension can be used as a classificator of urban land use [20]; we plan to explore this approach in our future research.

\section{Acknowledgement}

The authors acknowledge the support of the European Social Fund and the state budget of the Czech Republic (Project No. CZ.1.07/2.3.00/20.0170). The authors also acknowledge the support of the Internal Grant Agency of Palacký University in Olomouc (Project No. PrF_2013_024) 


\section{References}

[1] Batty M., Longley P.: Fractal Cities: A Geometry of Form and Function. Academic Press, London, 1994.

[2] Bromley O.: Star Fort, http://www.obromley.co.uk/web/concept/star_fort.htm, 2009.

[3] Chen Y., Jiang S.: An analytical process of the spatio-temporal evolution of urban systems based on allometric and fractal ideas. Chaos, Solitons \& Fractls, 39, 1, 2009, pp. 49-64.

[4] Chen Y:: Derivation of the functional relations between fractal dimension of and shape indices of urban form. Computers, Environment and Urban Systems, 35, 6, 2011, pp. 442451.

[5] De Keersmaecker M. L., Frankhauser P., Thomas I.: Using fractal dimension for characterizing intra-urban diversity: The example of Brussels. Paper presented at the ERSA 2003 Congress, Jyvaskyla, Finland, 27-30 September, 2003.

[6] Falconer K. J.: The Geometry of Fractal Sets. Cambridge University Press, Cambridge, 1985.

[7] Falconer K.: Fractal geometry: Mathematical foundations and applications. Chichester, John Wiley \& Sons. 1999.

[8] Ge M., Lin Q.: Realizing the Box-counting Method for Calculating Fractal Dimension of Urban Form Based on Remote Sensing Image. Geo-spatial Information Science (1 December 2009), doi: 10.1007/s11806-009-0096-1, 12, 4, 2009, pp. 265-270.

[9] Goodchild M. F.: Fractals and the accuracy of geographical measures. Math. Geol., 12, 1980, pp. 85-98.

[10] Mandelbrot B.: How Long Is the Coast of Britain? Statistical Self-Similarity and Fractional Dimension. Science, New Series, 156, 3775., doi:10.1126/science.156.3775.636, 1967, pp. 636638.

[11] Mandelbrot B.: The Fractal Geometry of Nature. W. H. Freeman. San Francisco, 1983.

[12] Marat-Mendez T., de Sampayo M. F., Rodrigues D. M. S.: Measuring Lisbon Patterns: Baixa from 1650 to 2010. Nexus Network Journal, 13, 2011, pp. 351-372.

[13] McMullen C. T.: Gallery, http://www.math.harvard.edu/ ctm/gallery/, 2009.

[14] Myint S. W.: Fractal approaches in texture analysis and classiffication of remotely sensed data: comparisons with spatial autocorrelation techniques and simple descriptive statistics. Int. J. Remote Sensing, 24, 9, 2003, pp. 1925-1947.

[15] Peitgen H. O., Jurgens H., Saupe D.: Fractals for the Classroom: Part 1: Introduction to Fractals and Chaos. Springer-Verlag, New York, 1992.

[16] Reynoso C.: The impact of chaos and complexity theories on spatial analysis - Problems and perspectives. Paper presented at the 24th Research Symposium: Reading Historical Spatial Information from around the World: Studies of Culture and Civilization Based on Geographic Information Systems Data, Kyoto, Japan, 7-11 February, 2005.

[17] Stanley H. E., Ostrosky N.: On Growth and Form: Fractal and Non-Fractal Patterns in Physics. Nijhoff, Boston, Mass., 1986.

[18] Sutherland S.: Mathematical Problem Solving with Computers: Fractal Dimension, 2002, http://www.math.sunysb.edu/ scott/Book331/Fractal_Dimension.html.

[19] Theiler J.: Estimating fractal dimension. J. Opt. Soc. Am. A, 7, 6, 1990, pp. 1055-1071.

[20] Thomas I., Tannier C., Frankhauser P.: Is there a link between fractal dimensions and other indicators of the built-up environment at a regional level. CyberGeo: European Journal of Geography, 13, 24, 2008. 